\title{
SHORT-TERM EFFECTS OF PHYSICAL THERAPY ON ADOLESCENT IDIOPATHIC SCOLIOSIS. A SERIES OF CASE STUDIES
}

\author{
Bogdan GOGA ${ }^{1^{*}}$ \\ ${ }^{1}$ National University of Physical Education and Sport, Faculty of Physical Therapy, Bucharest, Romania \\ *Corresponding author: bogdan.goga@ymail.com
}

https://doi.org/10.35189/dpeskj.2021.60.4.14

\begin{abstract}
Idiopathic scoliosis of the adolescent patient is one of the most common spinal deformities. Current studies on the effectiveness of conservative treatment do not provide sufficient data to confirm its validity, although the available literature proposes a multitude of nonsurgical treatments. Fifteen case studies were observed in a short period of conservative treatment based on the Schroth method combined with the Chêneau brace in order to highlight if stopping the progression of the disease or correcting the Cobb angle can be achieved during a growth period. The Cobb angle was measured at the beginning on the most recent X-ray, and after a period of six months, on a new X-ray. Thus, it was observed the short-term effect of therapeutic protocol. The physiotherapy programme consisted of five Schroth-based exercises, two muscle strength and endurance exercises and one auxiliary manual technique. Frequency was established at six times per week: three times in the rehabilitation clinic under the supervision of a Schroth physiotherapist and three times the patient applied the therapy at home, where each session lasted approximately 60 minutes. Limitations observed in the implementation of the programme were the level of involvement of the patient, the measurement of Cobb angle and the period of the year in which the programme was applied. Following these case studies, we believe that the benefits of this approach can create a basis for a guideline of conservative treatment, but further studies should be done with a higher number of people and with control and placebo groups.
\end{abstract}

Keywords: adolescent idiopathic scoliosis, Schroth method, Chêneau brace, Cobb angle.

\section{Introduction}

Scoliosis is a three-dimensional deformity of the spine and torso, which can deteriorate rapidly during periods of accelerated growth (Weiss \& Moramarco, 2013). It is characterised by abnormal curvatures in all three planes of movement, most notably in the frontal and horizontal planes. Approximately $80 \%$ of all cases of structural scoliosis are termed idiopathic, meaning that the condition has no apparent biological or mechanical cause (Van der Plaats et al., 2007). The remaining percentage (around 20\%) is caused by congenital abnormalities, neuromuscular pathologies, cerebral palsy, tumours, trauma or infectious diseases of the spine. Overall, about $2 \%$ to $3 \%$ of the adolescent population aged 10 to 16 years exhibits a lateral (frontal plane) curvature that exceeds 10 degrees (Lonstein, 2006). For unknown reasons, progressive idiopathic scoliosis affects adolescent females four times as often as males, especially those experiencing a rapid growth spurt (Roberto et al., 1997). When the balance fails, deformity typically occurs (Neumann, 2017).

Several theories have attempted to explain the cause of adolescent idiopathic scoliosis, including: natural subtle differences in bilateral symmetry of the body; uneven growth or abnormal histologic structure of connective tissue; asymmetry in paraspinal muscle activation 
and asymmetry in spinal loading, which leads to abnormal vertebral growth and intervertebral disc remodelling (Schlösser et al., 2014).

Despite widespread media, many physiotherapists and personal trainers believe that the biomechanical postural model cannot be considered the cause of this pathology and can only be taken into consideration as a risk factor for disease progression.

There are several classifications of scoliosis, and the chosen reference criterion is: aetiology, ability to be corrected, treatment, method of treatment or age. In terms of curvature reduction, scoliosis can be defined as functional or structural, where functional represents the capacity for correction with an active change in posture, and structural represents a fixed deformity that cannot be fully corrected with active or passive movements.

It is up to the paediatric orthopaedist to determine the indication and type of treatment. According to Stagnara's criteria, this specialist will decide the type of treatment: physical therapy if curvatures do not exceed a 30-degree Cobb angle, physical therapy combined with bracing if one curvature is greater than a 30-degree Cobb angle and surgery indication if the scoliosis deformity presents a Cobb angle above 50 degrees. These recommendations also depend on the skeletal maturity indicated by the Risser Sign. For example, a Risser Stage 5 patient will not have any clinical benefits from using a brace, although that person presents one or two curvatures greater than 30 degrees. On the other hand, a Risser Stage 0 patient who has a 27-degree curvature that has increased more than 5 degrees in the last six months can receive brace indication.

More attention will also be paid to the sagittal plane in the assessment of adolescent idiopathic scoliosis. The presence of a significantly reduced thoracic kyphosis justifies a stronger consideration for surgery, based on the probable ineffectiveness of bracing and the potential compromise of pulmonary function (Dreimann et al., 2014).

The complex treatment of adolescent idiopathic scoliosis aims at slowing down, stopping and even correcting a progressive curve and, in cases exceeding $30^{\circ}$, is composed of scoliosis-specific physiotherapy exercises and the Chêneau brace. Thus, the main objective of conservative treatment is to stop the progression and avoid surgery. The Schroth method has shown promising results in different studies (Park et al., 2018). The principle of treating idiopathic scoliosis using the German concept points out the following aspects: development of corrective postural behaviour during therapy and all daily living activities; treatment of the whole spine, not only the curvature; understanding and correcting weight bearing and patient education about deformity and aggravating factors.

The desired and ideal goal is to correct the curvature. The realistic goal of a therapeutic intervention is to stop the curve progression (Weiss \& Moramarco, 2013).

The research purpose (based on analysing case studies) is to reveal the role of scoliosisspecific physiotherapy exercises using the Schroth method combined with the Chêneau brace in the treatment of adolescent idiopathic scoliosis, as a basis for further and broader studies.

The research objective is to highlight the role of scoliosis-specific physiotherapy exercises and the Chêneau brace in the conservative treatment of adolescent idiopathic scoliosis. 


\section{Research question}

Can conservative treatment of idiopathic scoliosis using scoliosis-specific physiotherapy exercises based on the Schroth method and the Chêneau brace stop or even correct curvature progression in adolescent idiopathic scoliosis during the growth spurt period?

\section{Methodology}

\section{Participants}

The 15 case studies included patients who received indication for physiotherapy and the Chêneau brace, having all the diagnostic of adolescent idiopathic scoliosis. The therapeutic intervention took place at the "Terapie pentru Mișcare" (Therapy for Movement) Recovery Clinic between July 2020 and October 2020.

The following inclusion criteria were used:

- Adolescent scoliosis of unknown aetiology;

- Risser Sign 0 or 1;

- At least one curvature with a Cobb angle higher than $30^{\circ}$;

- A calculated risk of curvature progression more than $90 \%$;

- Absence of pain or paraesthesia;

- Chêneau brace.

Exclusion criteria:

- Adolescent scoliosis of known aetiology (e.g., congenital deformity of the vertebrae, cerebral palsy);

- Risser Sign 2, 3, 4 or 5;

- Cobb angle less than $30^{\circ}$ in curent curvatures;

- A calculated risk of curvature progression less than $90 \%$;

- Musculoskeletal pain or other symptoms;

- Brace of other type (Boston; EuroBoston; Milwaukee, etc.);

The data of the 15 participants are shown Table 1.

Table 1. Data of the participants

\begin{tabular}{lcccccc}
\hline Item no. & Initials & Age & Gender & Diagnosis & Functional diagnosis & Main curve \\
\hline 1 & A.M. & 13 & F & Adolescent idiopathic scoliosis & TriLle & thoracic \\
2 & A.S. & 12 & F & Adolescent idiopathic scoliosis & LriHleKt- & lumbar \\
3 & B.E. & 13 & F & Adolescent idiopathic scoliosis & LleTri & lumbar \\
4 & B.I. & 12 & F & Adolescent idiopathic scoliosis & LleTri & lumbar \\
5 & B.P. & 12 & F & Adolescent idiopathic scoliosis & LleTri & lumbar \\
6 & C.A. & 12 & F & Adolescent idiopathic scoliosis & LleKt- & lumbar \\
7 & C.B. & 13 & F & Adolescent idiopathic scoliosis & Tri & thoracic \\
8 & C.E. & 13 & F & Adolescent idiopathic scoliosis & LleKt- & lumbar \\
9 & D.C. & 12 & F & Adolescent idiopathic scoliosis & TriLle & thoracic \\
10 & I.P. & 13 & F & Adolescent idiopathic scoliosis & Tle & thoracic \\
11 & M.A. & 12 & F & Adolescent idiopathic scoliosis & TriHle & thoracic \\
12 & M.E. & 13 & F & Adolescent idiopathic scoliosis & TriLle & thoracic \\
13 & M.O. & 13 & F & Adolescent idiopathic scoliosis & LleTri & lumbar \\
\hline
\end{tabular}




\begin{tabular}{lllllcc}
\hline 14 & P.M. & 12 & F & Adolescent idiopathic scoliosis & LriKt- & lumbar \\
15 & T.S. & 12 & F & Adolescent idiopathic scoliosis & Tri & thoracic \\
\hline
\end{tabular}

The functional diagnostic corresponds to the Schroth method assessment classifications. The main author is a Schroth therapist certified in International Schroth Three-Dimensional Scoliosis Therapy - Asklepios - Katharina Schroth method.

\section{Instruments}

Children should be assessed on a regular basis every six months or maybe even a shorter period for back deformities. The most common clinical test for scoliosis screening is the Adams forward-bending test (Karachalios et al., 1999). If an asymmetry is observed, the clinician will refer the patient for a complete spine x-ray to confirm scoliosis. Depending on the x-ray result, the specialist will calculate the Cobb angle to define the magnitude of the curvature. The first mention of an angle that measures the magnitude of the scoliosis was in 1948 and was described by Dr John Cobb (Lonner et al., 2010). Thus, since then, the Cobb angle has been the standard for scoliosis assessment, representing the angle formed by the crossing lines drawn parallel to the neutral upper and lower vertebrae of each curvature (Cordun, 2009). A clinically relevant result for the reduction or progression of a curvature is a difference greater than 5 degrees Cobb Angle, a lower value being considered a calculation error (Lonner et al., 2010). Stagnara's criteria provide the means of indicating the appropriate therapeutic approach that the patient with adolescent idiopathic scoliosis will receive: between 0 and 30 degrees, physiotherapy; between 30 and 50 degrees, physiotherapy combined with bracing; above 50 degrees, indication for surgery. These results will also be taken into account for skeletal development, with the Risser Sign being used most often by clinicians as an indicator. The Risser Sign has 6 stages, each of them corresponding to a pubertal growth phase (Akbarnia et al., 2010). The clinician should take into consideration the risk of progression in relation to the growth potential of an individual in order to provide proper therapeutic indications, especially for patients with high progression risk (Weiss et al., 2018). The periods of accelerated growth represent timeframes in which the curvature is very likely to worsen, in comparison with the periods of slow growth when worsening is unlikely to occur (Hawes \& O'Brien, 2006). The lower the level of bone maturation, the higher the risk of progression is (Weinstein et al., 2003) and the greater the Cobb angle of the curvature is at the first diagnosis, the greater the risk of its aggravation (Lowe et al., 2000).

An example of Cobb angle measurement is shown in Figure 1. 


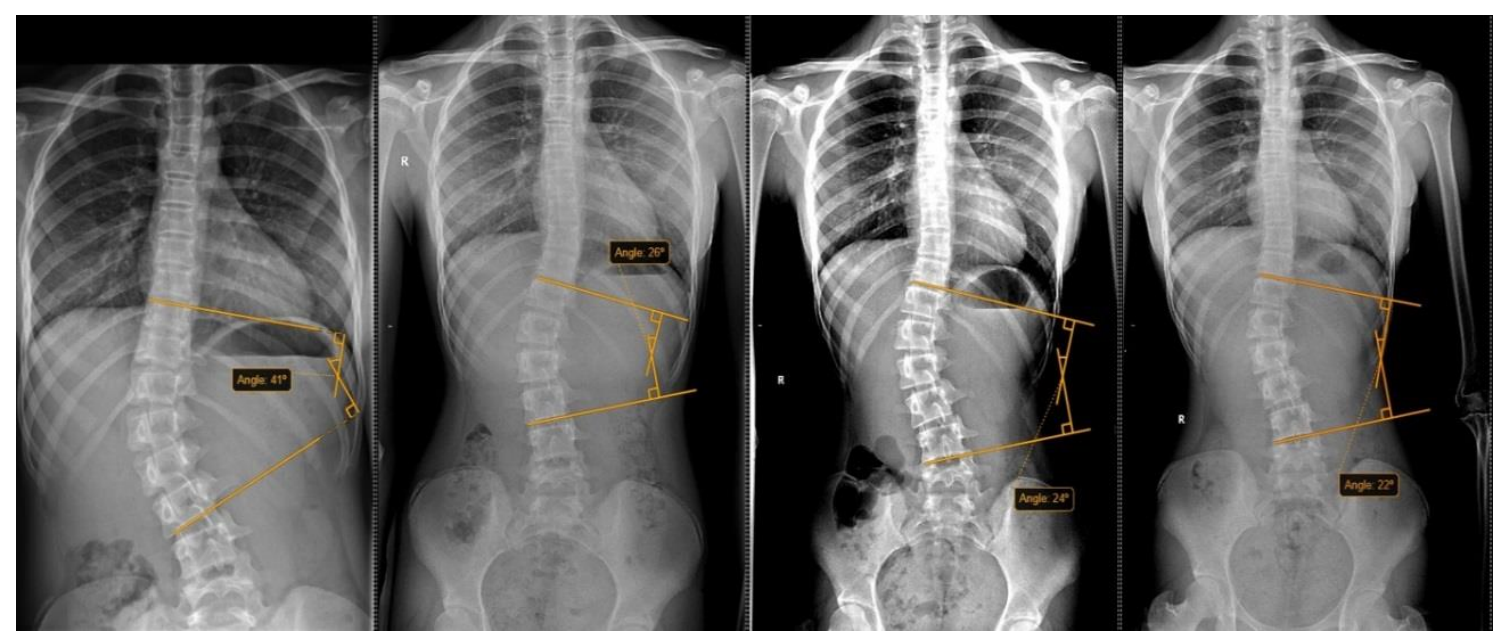

Figure 1. Example of Cobb angle measurement observed during two years of treatment

\section{Procedure}

Although scoliosis-specific exercises are used around the world, their overall relative effectiveness remains unproven (Romano et al., 2013).

Specific exercises will be performed in front of a mirror so that the patient receives constant feedback through the visual analyser. The therapist will also provide permanent voice feedback to help the patient better understand corrective and hypercorrective postures. The best of practice for this amount of feedback is to achieve it three-dimensionally. The exercises will be performed in a fundamental corrective isometric position that has to be maintained at least for 10 respiratory cycles.

The objects used in Schroth therapy are mirrors, rice bags, plastic cylinders, Schroth sticks, wall bars, gym mat, balls of different sizes and physio balls. Depending on the type and difficulty of the exercises, they are gradually introduced in order to be correctly performed and to avoid injuries.

From the beginning, the goal is to make the patient understand the concept of correction of the centre of gravity in standing, sitting or daily living activity positions. Due to the fact that weight bearing is generally on the lower limb adjacent to the convexity of the main curve, the therapist will provide education about shifting it on both legs.

Angular breathing is a specific Schroth-method pattern aimed at activating inhalation and exhalation. Rib humps will be maintained isometrically during the maximum expiratory movement, and the depressions will be more pronounced in the maximum inspiratory rib positions to help correct trunk asymmetry.

When the physiotherapist assesses the patient and confirms that they are able to actively execute Schroth corrections, understand corrective weight bearing and activate angular breathing, the design of the therapeutic exercise will start. The exercise will performed by maintaining isometrically all specific corrections applied to the Schroth functional diagnostic and carrying out 4-5 respiratory cycles (as a beginner) up to 10 respiratory cycles. Experienced well-trained patients can reach the performance of doing an exercise for 5 minutes without any break. 
Scoliosis-specific physiotherapy exercises (based on Schroth) consist of five Schroththerapy exercises, five general exercises and two auxiliary manual techniques adapted to each patient, depending on the main curvature, plus two strengthening exercises. The programme includes a first series of five daily sessions, followed by three sessions per week up to six months in the clinic with a Schroth physiotherapist, plus three home sessions per week under the supervision of a parent. Each session lasts for 50 minutes. The programme is carried out over a period of at least 6 months.

- Exercise 1: Symmetric and asymmetric muscle cylinder from standing (Figure 2);

- Exercise 2: Lateral muscle cylinder;

- Exercise 3: Big Bow done on the gymnastics wall bars;

- Exercise 4: Standing cylinder using two Schroth sticks;

- Exercise 5: Prone contraction of the shoulder;

- Mobilisation technique: Posterior hip depression according to PNF pelvis patterns for primary lumbar curvature or de-rotation of the posterior rib hump for primary thoracic curve.

Each exercise was performed in 4 sets of 10 respiratory cycles. These exercises represent parts of the Schroth method and are well known by any specialist trained according to the Asklepios Katharina Schroth method.

- High plank: Maintaining Schroth corrections (on palms) according to the functional diagnostic - 2 sets of 1 minute.

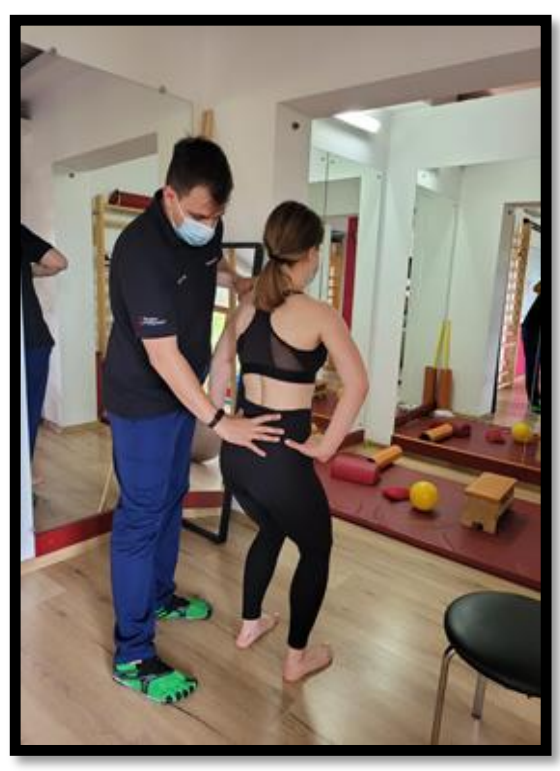

Figure 2. Standing symmetric muscle cylinder

The need for a device to stop the progression of scoliosis and correct it has been a challenge since ancient times. Bracing is applied at a Cobb angle greater than 30 degrees, according to Stagnara's criteria. In the modern era, a variety of corsets are mostly used depending on the geographical area where specialists work rather than due to their therapeutic effectiveness. Zaina et al. (2014) list a number of braces depending on the 
geographical area where they are recommended. Thus, on the European continent, Chêneau braces and their derivatives seem to be the most common, a trend also observed in Romania.

The Chêneau brace is an orthosis made by a thermoplastic process on a plastered hypercorrective mould taken from the patient. The general principle of correction is that of distortion and normalisation of the sagittal plane, which would correct the coronary and transverse planes, resulting in some elongation of the spine without any force of significant distraction (Rigo, 1999). Braces play an important role and a scientifically proven place in the treatment of scoliosis, as they can significantly decrease the progression of curves with high surgical risk in patients with adolescent idiopathic scoliosis (Weinstein et al., 2013).

The indication for wearing the brace is 22-23 hours per day. The rest of the time is allocated to physiotherapy and personal hygiene. The Chêneau brace has similar principles to the Schroth method, with pressures applied to the rib humps and open zones for the expansion of depressions. Lower levels of compliance and involvement in the physiotherapy programme and wearing the brace are critical aspects, being key parts of therapeutic success.

A Chêneau brace from personal practice is shown in Figure 3.

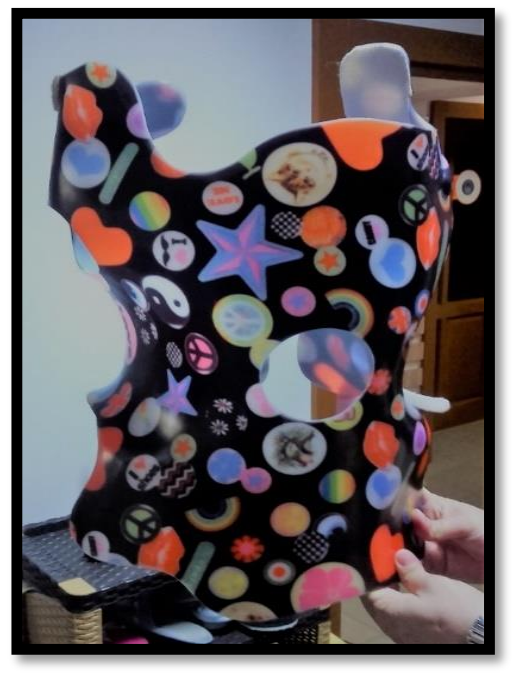

Figure 3. A Chêneau brace from personal practice

\section{Results}

Given the long period in which the treatment takes place, the level of compliance and involvement in therapeutic solutions can influence the final results. Thus, we emphasise the need to create clearer strategies for acceptance and compliance with the physiotherapy programme and wearing the brace, as it is a medium- and long-term programme. Currently, there is no clear direction or procedural guidance in this regard.

Participants' Cobb angles measured initially and after 6 months are shown in Table 2. 
Table 2. Cobb angles measured initially and after 6 months

\begin{tabular}{|c|c|c|c|c|c|}
\hline \multirow{2}{*}{ Item no. } & \multirow{2}{*}{ Initials } & \multicolumn{2}{|c|}{ Initial Cobb angle (degrees) } & \multicolumn{2}{|c|}{ Cobb angle after 6 months (degrees) } \\
\hline & & thoracic (T) & lumbar $(\mathrm{L})$ & thoracic $(\mathrm{T})$ & lumbar (L) \\
\hline 1 & A.M. & 35 & 25 & 30 & 10 \\
\hline 2 & A.S. & 0 & 26 & 0 & 24 \\
\hline 3 & B.E. & 70 & 69 & 62 & 61 \\
\hline 4 & B.I. & 43 & 43 & 40 & 41 \\
\hline 5 & B.P. & 31 & 40 & 30 & 36 \\
\hline 6 & C.A. & 35 & 0 & 15 & 00 \\
\hline 7 & C.B. & 30 & 0 & 28 & 0 \\
\hline 8 & C.E. & 55 & 0 & 38 & 0 \\
\hline 9 & D.C. & 30 & 0 & 30 & 0 \\
\hline 10 & I.P. & 42 & 0 & 28 & 0 \\
\hline 11 & M.A. & 50 & 0 & 43 & 0 \\
\hline 12 & M.E. & 38 & 25 & 35 & 30 \\
\hline 13 & M.O. & 54 & 41 & 54 & 41 \\
\hline 14 & P.M. & 30 & 0 & 20 & 0 \\
\hline 15 & T.S. & 37 & 0 & 27 & 0 \\
\hline
\end{tabular}

a) Thoracic Cobb angle

Table 3. Thoracic Cobb angle

\begin{tabular}{lcccccccc}
\hline \multicolumn{2}{c}{ Testing } & $\begin{array}{c}\text { Mean } \\
\text { difference }\end{array}$ & Median & $\begin{array}{c}\text { Standard } \\
\text { deviation }\end{array}$ & Min & Max & Range & $\begin{array}{c}\text { Coefficient of } \\
\text { variation }\end{array}$ \\
\hline Initial & 38.67 & -6.67 & 37 & 15.7 & 0 & 70 & 70 & $40.6 \%$ \\
Final & 32.00 & & 30 & 15.0 & 0 & 62 & 62 & $46.8 \%$ \\
\hline
\end{tabular}

The mean value of degrees for thoracic Cobb angles measured in the assessment process of patients with idiopathic scoliosis aged 12-14 decreased by 6.67 degrees, from 38.67 in the initial test to 32.00 degrees in the final test (Table 3). The mean difference is within the range $(-10.23$; -3.10) in proportion of $95 \%$. The degree of dispersion of Cobb angles is inhomogeneous in both tests.

Table 4. Statistical indicators for the bilateral t-test (thoracic)

\begin{tabular}{lccccccc}
\hline & \multicolumn{2}{c}{ Results of statistical indicators (TF-TP) } & & \multicolumn{3}{c}{ Bilateral t-test } \\
Average & $\begin{array}{c}\text { Standard } \\
\text { deviation }\end{array}$ & $\begin{array}{c}\text { Standard } \\
\text { error }\end{array}$ & $\begin{array}{c}95 \% \text { confidence } \\
\text { interval }\end{array}$ & Effect size & t & df & $p$ \\
\hline-6.67 & 6.43 & 1.66 & $(-10.23 ;-3.10)$ & 1.04 & 4.01 & 14 & 0.001 \\
\hline
\end{tabular}

The bilateral t-test showed a statistically significant difference in averages, $p=0.001<$ 0.05 , for $\mathrm{t}=4.01$ and $\mathrm{df}=14$. The effect size (1.04) indicates a very large difference between the averages. (Table 4) 
Table 5. Thoracic Cobb angle after 6 months

\begin{tabular}{cccc}
\hline Mean difference $(\mathrm{F}-\mathrm{I})$ & Progress & Difference size & The progress is \\
\hline-6.67 & $17.2 \%$ & Significant & Statistically significant \\
\hline
\end{tabular}

There is a significant decrease in the average thoracic Cobb angle after 6 months of applying the physiotherapy programme based on Schroth principles (Table 5). The graphical representation of the degrees of average Cobb angles in the two tests, the values of initial and final Cobb angles for each patient and their differences are shown in Figure 4 and Figure 5.

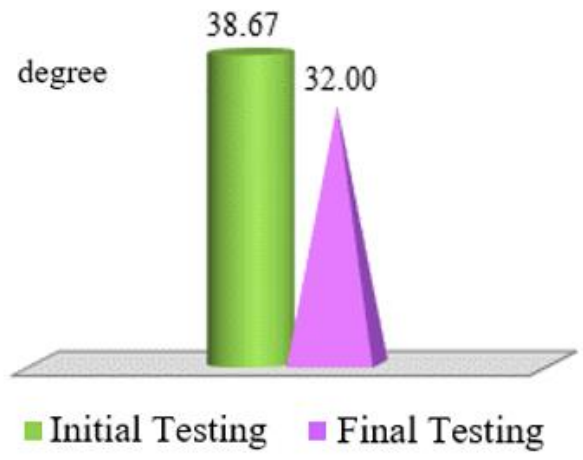

Figure 4. Average thoracic values

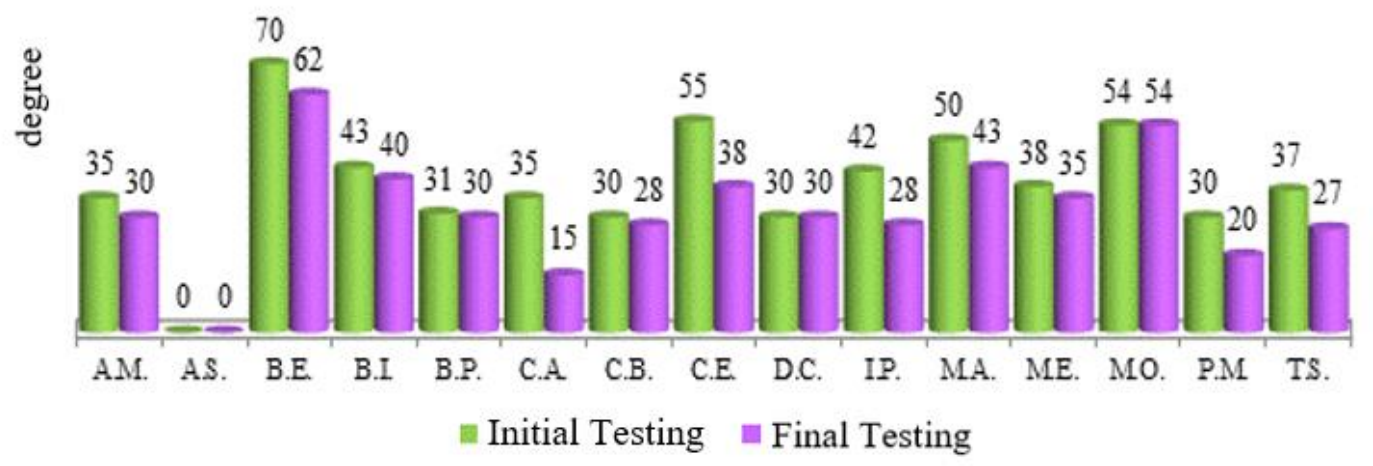

Figure 5. Initial and final measurements of thoracic curves

b) Lumbar Cobb angle

Table 6. Lumbar Cobb angle

\begin{tabular}{lcccccccc}
\hline \multicolumn{2}{c}{ Testing } & $\begin{array}{c}\text { Mean } \\
\text { difference }\end{array}$ & Median & $\begin{array}{c}\text { Standard } \\
\text { deviation }\end{array}$ & Min & Max & Range & $\begin{array}{c}\text { Coefficient } \\
\text { of variation }\end{array}$ \\
\hline Initial & 17.93 & -1.73 & 0 & 22.3 & 0 & 69 & 69 & $124.6 \%$ \\
Final & 16.20 & & 0 & 20.7 & 0 & 61 & 61 & $128.0 \%$ \\
\hline
\end{tabular}

The average Cobb angles determined in the assessment of idiopathic lumbar scoliosis for patients aged 12-14 decreased by 1.73 degrees, from 17.93 in the initial test to 16.20 degrees in the final test (Table 6). The mean difference is within the confidence interval $(-4.27 ; 0.80)$. The degrees of Cobb angles are not homogeneously dispersed in any of the two tests. 
Table 7. Statistical indicators for the bilateral t-test (lumbar)

\begin{tabular}{cccccccc}
\hline & \multicolumn{2}{c}{ Results of statistical indicators (TF-TP) } & \multicolumn{4}{c}{ Bilateral t-test } \\
Average & $\begin{array}{c}\text { Standard } \\
\text { deviation }\end{array}$ & $\begin{array}{c}\text { Standard } \\
\text { error }\end{array}$ & $\begin{array}{c}\text { 95\% confidence } \\
\text { interval }\end{array}$ & Effect size & $\mathrm{t}$ & $\mathrm{df}$ & $\mathrm{P}$ \\
\hline-1.73 & 4.57 & 1.18 & $(-4.27 ; 0.80)$ & 0.38 & 1.47 & 14 & 0.164 \\
\hline
\end{tabular}

The mean difference is statistically insignificant according to the bilateral dependent t-test, where $\mathrm{p}=0.164>0.05$, for $\mathrm{t}=1.47$ and $\mathrm{df}=14$. The effect size (0.38) shows a small-tomedium difference between the averages. (Table 7)

Table 8. Lumbar Cobb angle after 6 months

\begin{tabular}{cccc}
\hline Mean difference (F-I) & Progress & Difference size & The progress is \\
\hline-1.73 & $9.7 \%$ & Small to medium & Statistically insignificant \\
\hline
\end{tabular}

There was no significant decrease in mean lumbar Cobb angle after 6 months of physical therapy based on Schroth principles (Table 8). The graphical representation of the degrees of average Cobb angles in the two tests, the values of initial and final Cobb angles for each patient and their differences are shown in Figure 6 and Figure 7.

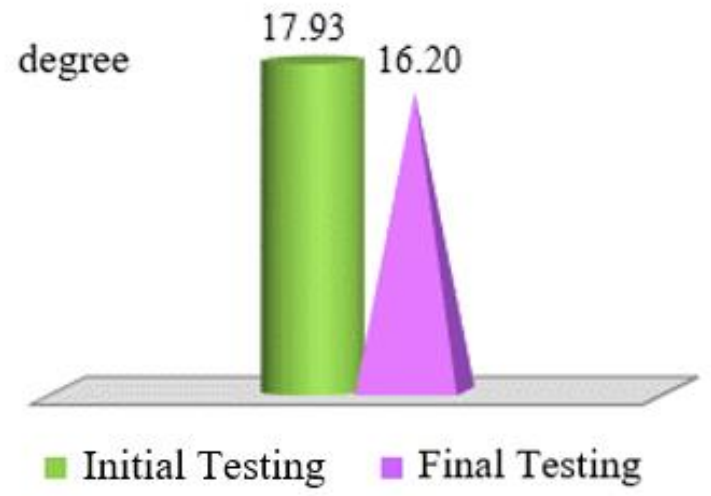

Figure 6. Average lumbar values

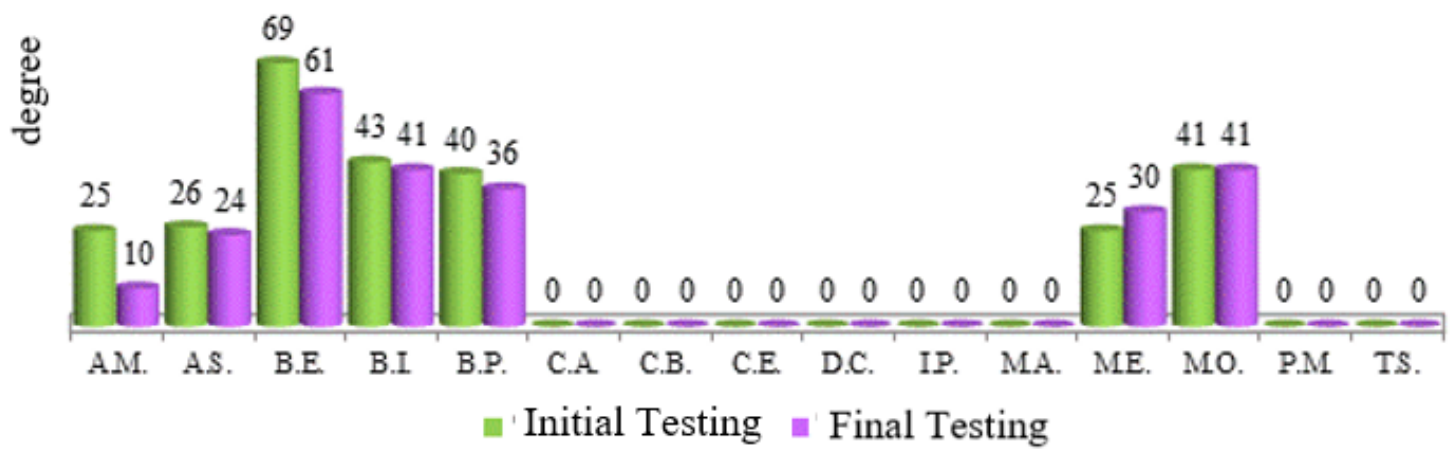

Figure 7. Initial and final measurements of lumbar curves 
Short-term results can be the basis for further research to the point where patients reach bone maturation (Risser 5 and 2 and a half year - 3 years after the onset of menstruation in women).

The average Cobb angle decreased in lumbar curvature, but not statistically significantly. A larger cohort study is indicated to be able to see whether there is a difference in clinical manifestation and progression between the two types of curves.

The fact that there are differences in the scoliosis development between thoracic curves and lumbar curves can represent a basis for a future research in this direction.

\section{Conclusion}

The results obtained through the specific physiotherapy programme based on Schroth principles combined with the intensive use of the Chêneau brace show that conservative treatment can stop the progression of adolescent idiopathic scoliosis in a short period of time.

Schroth therapy should become the form of physiotherapy of choice in the treatment of adolescent idiopathic scoliosis, and physiotherapists should specialise in it to work with such patients. This could be developed as part of a future master's programme in paediatric physiotherapy.

It is necessary to create strategies for acceptance and compliance with the physiotherapy programme and wearing the brace, as it is a medium- and long-term programme.

Evidence-based practice becomes step by step the activity of the physiotherapist, who acquires new skills and advances from a performer to a practitioner who can develop and support a clinical reasoning adapted to each patient.

The results could be the basis for a comparative study with other physiotherapy methods or a control group in order to develop clear guidelines for the conservative treatment of adolescent idiopathic scoliosis.

\section{References}

Akbarnia, B. A., Yazici, M., \& Thompson, G., H. (2010). The growing spine management of spinal disorders in young children. Springer.

Cordun, M. (2009). Kinantropometrie [Kinanthropometry]. CD Press.

Dreimann, M., Hoffmann, M., Kossow, K., Hitzl, W., Meier, O., \& Koller, H. (2014). Scoliosis and chest cage deformity measures predicting impairments in pulmonary function: A cross-sectional study of 492 patients with scoliosis to improve the early identification of patients at risk. Spine, 39(24), 2024-2033. https://doi.org/10.1097/brs.0000000000000601

Hawes, M. C., \& O'Brien, J. P. (2006). The transformation of spinal curvature into spinal deformity: pathological processes and implications for treatment. Scoliosis, 1(1): 3. https://doi.org/10.1186/1748-7161-1-3

Karachalios, T., Sofianos, J., Roidis, N., Sapkas, G., Korres, D., \& Nikolopoulos, K. (1999). Ten-year follow-up evaluation of a school screening program for scoliosis. Is the forwardbending test an accurate diagnostic criterion for the screening of scoliosis? Spine, 24(22), 2318-2324. https://doi.org/10.1097/00007632-199911150-00006 
Lonner, B. S., Auerbach, J. D., Sponseller, P., Rajadhyaksha, A. D., \& Newton, P. O. (2010). Variations in pelvic and other sagittal spinal parameters as a function of race in adolescent idiopathic scoliosis. Spine, 35(10), E374-E377. https://doi.org/10.1097/brs.0b013e3181bb4f96

Lonstein, J. E. (2006). Scoliosis: Surgical versus nonsurgical treatment. Clinical Orthopaedics and Related Research, 443, 248-259. https://doi.org/10.1097/01.blo.0000198725.54891.73

Lowe, T. G., Edgar, M., Margulies, J. Y., Miller, N. H., Raso, V. J., Reinker, K. A., \& Rivard, C. H. (2000). Etiology of idiopathic scoliosis: Current trends in research. The Journal of Bone and Joint Surgery, 82(8), 1157-1168. https://doi.org/10.2106/00004623-200008000-00014

Neumman, D. A. (2017). Kinesiology of the musculoskeletal system: Foundations for rehabilitation (3rd ed.). Elsevier, Inc.

Park, J.-H., Jeon, H.-S., \& Park, H.-W. (2018). Effects of the Schroth exercise on idiopathic scoliosis: A meta-analysis. European Journal Physical and Rehabilitation Medicine, 54(3), 440-449. https://doi.org/10.23736/s1973-9087.17.04461-6

Rigo, M. (1999). 3D correction of trunk deformity in patients with idiopathic scoliosis using Chêneau brace. In Studies in health technology and informatics (Vol. 59: Research into spinal deformities 2, pp. 362-365). DOI: 10.3233/978-1-60750-903-5-362

Roberto, R. F., Lonstein, J. E., Winter, R. B., \& Denis, F. (1997). Curve progression in Risser stage 0 on patients after posterior spinal fusion for idiopathic scoliosis. Journal of Pediatric Orthopaedics, 17: 718. https://doi.org/10.1097/00004694-199711000-00005

Romano, M., Minozzi, S., Zaina, F., Bettany-Saltikov, J., Chockalingam, N., Kotwicki, T., Maier-Hennes, A., \& Negrini, S. (2013). Exercises for adolescent idiopathic scoliosis: A Cochrane systematic review. Spine, 38(14), E883-E893.

https://doi.org/10.1097/brs.0b013e31829459f8

Schlösser, T. P. C., Van Stralen, M., Brink, R. C., Chu, W. C. W., Lam, T.-P., Vincken, K. L., Castelein, R. M., \& Cheng, J. C. Y. (2014). Three-dimensional characterization of torsion and asymmetry of the intervertebral discs versus vertebral bodies in adolescent idiopathic scoliosis. Spine, 39(19), E1159-E1166. https://doi.org/10.1097/brs.0000000000000467

Van der Plaats, A., Veldhuizen, A. G., \& Verkerke, G. J. (2007). Numerical simulation of asymmetrically altered growth as initiation mechanism of scoliosis. Annual Biomedical Engineering, 35(7), 1206-1215. https://doi.org/10.1007/s10439-007-9256-3

Weinstein, S. L., Dolan, L. A., Spratt, K. F., Peterson, K. K., Spoonamore, M. J., \& Ponseti, I. V. (2003). Health and function of patients with untreated idiopathic scoliosis: a 50-year natural history study. JAMA, 289(5), 559-567. https://doi.org/10.1001/jama.289.5.559

Weinstein, S. L., Dolan, L. A., Wright, J. G., \& Dobbs, M. B. (2013). Effects of bracing in adolescents with idiopathic scoliosis. New England Journal of Medicine, 369(16), $1512-$ 1521. https://doi.org/10.1056/nejmoa1307337

Weiss, H. R., \& Moramarco, M. (2013). Scoliosis - treatment indications according to current evidence. Musculoskeletal Medicine, 1(1): 1. https://doi.org/10.13172/2052-9287-1-1-347

Weiss, H. R., Lehnert-Schroth, C., Moramarco, M., \& Moramarco, K. (2018). Schroth therapy: Advancements in conservative scoliosis treatment (2nd ed.). Lap Lambert Academic Publishing.

Zaina, F., De Mauroy, J. C., Grivas, T., Hresko, M. T., Kotwizki, T., Maruyama, T., Price, N., Rigo, M., Stikeleather, L., Wynne, J., \& Negrini, S. (2014). Bracing for scoliosis in 2014: State of the art. European Journal of Physical Rehabilitation Medicine, 50(1), 93110. PMID: 24622051 